\title{
CELT site testing program
}

Matthias Schoeck, D. Andre Erasmus, S. George Djorgovski, Gary A. Chanan, Jerry E. Nelson

Matthias Schoeck, D. Andre Erasmus, S. George Djorgovski, Gary A. Chanan, Jerry E. Nelson, "CELT site testing program," Proc. SPIE 4840, Future Giant Telescopes, (30 January 2003); doi: 10.1117/12.459124

Event: Astronomical Telescopes and Instrumentation, 2002, Waikoloa, Hawai'i, United States 


\title{
CELT site testing program
}

\author{
Matthias Schöck $^{a}$, D. André Erasmus ${ }^{b}$, S. George Djorgovski ${ }^{c}$, Gary A. Chanan $^{a}$, \\ and Jerry E. Nelson ${ }^{d}$ \\ ${ }^{a}$ University of California, Irvine, CA, USA \\ ${ }^{b}$ South African Astronomical Observatory, Observatory 7935, South Africa \\ ${ }^{c}$ California Institute of Technology, Pasadena, CA, USA \\ ${ }^{d}$ University of California, Santa Cruz, CA, USA
}

\begin{abstract}
The California Extremely Large Telescope, CELT, is a proposed 30-m telescope. Choosing the best possible site for CELT is essential in order to extract the best science from the observations and to reduce the complexity of the telescope. Site selection is therefore currently one of the most critical pacing items of the CELT project. In this paper, we first present selected results from a survey of the atmospheric transparency at optical and infrared wavelengths over the southwestern USA and northern Mexico using satellite data. Results of a similar study of South America have been reported elsewhere. These studies will serve as the pre-selection criterion of the sites at which we will perform on-site testing. We then describe the current status of on-site turbulence evaluation efforts and the future plans of the CELT site testing program.
\end{abstract}

Keywords: Extremely large telescopes, site selection, atmospheric turbulence characterization, satellite remote sensing

\section{THE CALIFORNIA EXTREMELY LARGE TELESCOPE, CELT}

The California Extremely Large Telescope, CELT, is a project being developed by the University of California (UC) and the California Institute of Technology (CIT) with the objective to build a telescope with a 30-meter, fully steerable aperture. Some of the key design features are a 30-meter filled aperture with more than 1000 individual segments, a 20 arc minute field of view (in seeing-limited mode of operation), and a wavelength coverage from the near ultraviolet to the mid infrared $(0.3-30 \mu \mathrm{m})$. Design specifications for adaptive optics call for a Strehl ratio of 0.5 at $1 \mu \mathrm{m}$ which will be realized by a multi-conjugate adaptive optics system optimized for the $1-2.5 \mu \mathrm{m}$ range. ${ }^{1-4}$ In order to obtain the highest-quality observations and to reduce the complexity of the telescope, it is essential that CELT will be located at the best possible site. In this paper, we describe the current status of our effort to find such a site. We present details of the satellite studies used to pre-select a site and of our plans for on-site testing of atmospheric turbulence conditions.

\section{CELT SITE SELECTION CRITERIA}

Site selection is a fundamental issue for the construction of a telescope of any size, but in particular for the coming generation of "extremely large" telescopes (ELTs), a term by which one usually refers to telescopes larger than the currently existing telescopes in the 8-10 meter range. Obviously, the fraction of usable nights, determined for the most part by the weather, that is, the cloud cover and the precipitable waver vapor (PWV) at the site, is the lowest-order and most important selection criterion. The creation of a short-list of sites based on this criterion is therefore the first step in the CELT site selection effort. Satellite studies of the cloud cover and PWV of northern Chile ${ }^{5}$ [funded by the Association of Universities for Research in Astronomy (AURA) and the University of Tokyo (UT) with support from European Southern Observatory (ESO)] and southwestern North America [funded by AURA and CELT with contribution of data partially funded by UT] have been performed and a comparative study between Chile, southwestern North America, and Hawaii funded by AURA

Further author information: (Send correspondence to M.S.)

M.S.: E-mail: mschoeck@uci.edu 
and CELT] will follow. All these studies will be taken into account for the CELT site decision. As an example, a detailed description of the North America study will be given in Section 3.

Another quantity falling into the weather category is the ground wind speed which is of great importance for the structural design of an ELT. Measurements of the ground wind speed are routinely taken at existing observatory sites so that data bases for these sites exist. In addition, weather stations have been installed by AURA at a few selected sites in Chile and more such stations will be deployed at other locations in a collaboration between CELT and AURA.

As mentioned in Section 1, the quality of atmospheric turbulence it of extreme importance for the design (and later for the operation) of CELT and will eventually determine the science that can be done with CELT. The basics of atmospheric turbulence characterization as they apply to ELTs and the current plans for on-site testing for CELT are described in Section 4.

Although weather and turbulence are arguably the most important factors for the CELT site selection process, there are many other issues that need to be taken into account. These include but are not limited to the seismic and volcanic activity at and around the site, political and and sociological issues such as availability, security, and staffing, and practical concerns such as logistics and cost of construction and operation. All these factors need to be taken into account in the final site decision for CELT. They are, however, not discussed in this paper.

\section{SATELLITE SURVEY OF CLOUD COVER AND WATER VAPOR IN THE SOUTHWESTERN U.S.A. AND NORTHERN MEXICO}

\subsection{Overview of the study}

A study to survey cloud cover and water vapor over the Southwestern U.S.A. and Northern Mexico using satellite data was funded by the Association of Universities for Research in Astronomy (AURA) and CELT, and used data that were partially funded by the University of Tokyo. ${ }^{6}$ In this survey, five years of satellite data from the Geostationary Operational Environmental Satellite (GOES) - East (Nadir: $0^{\circ}, 75^{\circ} \mathrm{W}$ ) were used to map the aerial distribution of cloud cover and precipitable water vapor (PWV) over the area of interest.

Meteorological satellite observations provide a consistent measurement over a wide field of view so that an objective comparison of sub-regions can be made. The spatial $(9.1 \mathrm{~km} \times 8.0 \mathrm{~km}$ at Nadir) and temporal $(3$ hours) resolution of the observations also allows for the characterization of conditions for particular sites at night or during daylight hours.

The satellite data used in the survey consisted of $6.7-\mu \mathrm{m}$ (water vapor channel) and 10.7- $\mu \mathrm{m}$ (IR window channel) imagery from the International Satellite Cloud Climatology Project (ISCCP) data set. The data covered the period July 1, 1993 to February 28, 1996 and June 1, 1997 to August 31, 1999. The data for the study area $\left(18^{\circ} \mathrm{N}\right.$ to $40^{\circ} \mathrm{N}$ and $96^{\circ} \mathrm{W}$ to $\left.124^{\circ} \mathrm{W}\right)$ were extracted from the full earth scans. Sample images of the study area are shown in Figure 1.

In order to determine the presence of cloud in the IR window imagery and to compute an absolute humidity from the satellite-derived upper tropospheric humidity (UTH), a measurement of the temperature profile above the ground is required. This is obtained from rawinsonde data. At several locations within the study area, balloons carrying instrument packages are released between two (00UT and 12UT) and four (additionally 06UT and 18UT) times per day. These data were obtained on CD from the National Climatic Data Center (NCDC). Data from 44 stations over the study area were used for this purpose.

To map cloud cover, information on terrain height is required. Digital terrain heights at 30 arc seconds $(\approx 1 \mathrm{~km})$ resolution were obtained from the United States Geological Survey (http://edcdaac.usgs.gov/ gtopo30/gtopo30.html). These data were processed to accentuate higher terrain by setting the terrain height for a given point equal to the maximum height of the 36 closest terrain points (a $5 \mathrm{~km} \times 5 \mathrm{~km}$ square). 

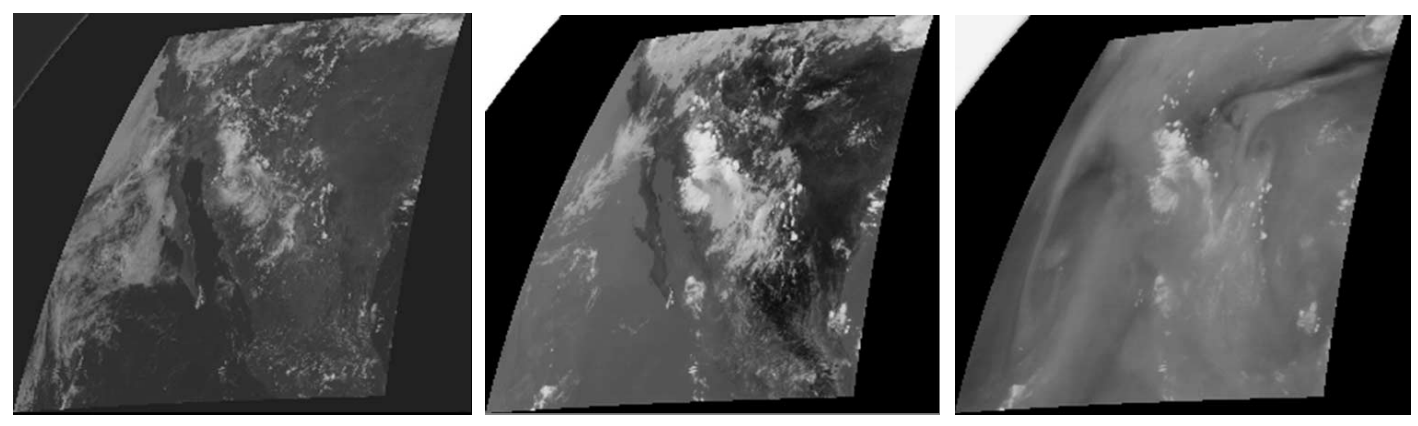

Figure 1. Sample ISCCP satellite images sectors of the study area $\left(18^{\circ} \mathrm{N}\right.$ to $40^{\circ} \mathrm{N}$ and $96^{\circ} \mathrm{W}$ to $\left.124^{\circ} \mathrm{W}\right)$ on $\mathrm{March} 20$, 1998 at $0845 \mathrm{UT}$. From left to right: visible channel $(0.55 \mu \mathrm{m})$, infra-red window channel $(10.7 \mu \mathrm{m})$ and water vapor channel $(6.7 \mu \mathrm{m})$.

\subsection{Methodology}

Measurements of cloud cover and water vapor were derived from satellite observations at $10.7 \mu \mathrm{m}$ in the infrared window and at $6.7 \mu \mathrm{m}$, a water vapor absorption band. In the IR window channel, emissions reach the satellite largely unattenuated by the atmosphere so that radiance values measured are due to emission from the surface. However, if clouds are present, they behave as elevated emitting "surfaces" so that radiation reaching the satellite is from the cloud top. Observations at $6.7 \mu \mathrm{m}$ are used to detect the presence and thickness of high altitude $(9-12 \mathrm{~km})$ cirrus clouds and, under clear conditions, to measure water vapor resident in the middle and upper troposphere.

True radiance values are obtained from the channel counts using a bias scaling factor, $b$, and a first order gain scaling factor, $m$, supplied in the calibration data as follows

$$
R=\frac{X-b}{m}
$$

where $R$ is radiance $\left(\mathrm{mW} /\left[\mathrm{m}^{2} \mathrm{sr} \mathrm{cm}-1\right]\right)$ and $\mathrm{X}$ is the count value. The brightness (or effective) temperature is then obtained by inverting the Planck function as follows

$$
T_{e f f}=\frac{c_{2} \nu}{\ln \left(1+\left[c_{1} \nu^{3}\right] / R\right)}
$$

where $T_{\text {eff }}$ is effective temperature $(\mathrm{K})$, "ln" stands for natural logarithm and $\nu\left(\mathrm{cm}^{-1}\right)$ is the central wave number of the channel. The coefficients $c_{1}$ and $c_{2}$ are the two radiation constants and have values of $c_{1}=$ $1.191066 \cdot 10^{-5}\left(\mathrm{~mW} \mathrm{~m}^{-2} \mathrm{sr}^{-1} \mathrm{~cm}^{4}\right)$ and $c_{2}=1.438833(\mathrm{~cm} \mathrm{~K})$. To convert effective temperature to actual temperature $T(\mathrm{~K})$, the following formula is used

$$
T=b T_{e f f}+a .
$$

The constants $a(\mathrm{~K})$ and $b$ depend on the observation channel. These are bias and gain adjustments that account for variations in the inverse Planck function across the spectral passband of the channel. The differences between the values of $T$ and $T_{\text {eff }}$ increase with decreasing temperature. They are usually of the order of $0.1 \mathrm{~K}$ and hence negligible for most calculations.

Using Eqs. (1) - (3) two temperatures $\left(T_{i r}\right.$ and $T_{w v}$ ) are computed, one for each observation channel. For GOES data, Soden and Bretherton ${ }^{7,8}$ have derived a semi-empirical relationship between $T_{w v}$ and the Upper Tropospheric Humidity (UTH) which is the mean relative humidity of the layer between $600 \mathrm{mb}$ and $300 \mathrm{mb}$. Using an independent upper-air temperature measurement from the rawinsonde an absolute humidity is derived from the UTH which, in turn, enables computation of the precipitable water vapor (PWV) above a given 
altitude or pressure level. Details on the computation of PWV are not provided here since the results presented in Section 3.3 of this paper will be limited to cloud cover.

The presence of cirrus (high altitude) clouds and their thickness is inferred from the $6.7 \mu \mathrm{m}$ imagery. Since these clouds are found at an altitude $(9-12 \mathrm{~km})$ higher than the water vapor emission layer, IR radiation from water vapor below the $300-\mathrm{mb}$ level $(\approx 9 \mathrm{~km})$ is absorbed and re-emitted at colder temperatures by the cloud particles. When UTH values rise to around $50 \%$, cirrus cloud particles start forming by condensation and deposition (the cloud particles may not be visible at this stage or have any effect on optical transparency). As UTH values rise further, the cloud particles grow in size and number and the cirrus cloud gets thicker, eventually becoming opaque. The relationship between cirrus cloud transparency and UTH has been defined in Refs. 9 and 10.

The 10.7- $\mu \mathrm{m}$ channel data are used to detect cloud in the middle and upper troposphere. Pixel temperatures $\left(T_{i r}\right)$ computed from the $10.7-\mu \mathrm{m}$ satellite data were compared to a reference temperature $\left(T_{r}\right)$ derived from the rawinsonde. Typically, temperature drops with height in the atmosphere, so if $T_{i r}$ is colder (by some margin) than the reference temperature at a given pixel location, the presence of cloud is indicated.

The cloud detection reference temperature $\left(T_{r}\right)$ is computed by estimating the surface pressure $\left(P_{s}\right)$ and corresponding surface temperature $\left(T_{s}\right)$ for a given pixel location using the rawinsonde data and the terrain height. Given the altitude of the surface and the geopotential heights of the pressure levels, $P_{s}$ and $T_{s}$ can be estimated by interpolation from the rawinsonde data. However, the actual surface temperature may be warmer or colder than $T_{s}$ since there is usually additional cooling (night) or warming (day) of the air by contact with the ground. If the estimated surface temperature is cooler than the actual surface temperature (day-time) cloud detection is not compromised. However, if the actual temperature is colder than the estimated surface temperature $\left(T_{s}\right)$ and $T_{i r}<T_{r}=T_{s}$, then two conditions are possible - cloud may be present or the ground is cold and is incorrectly being interpreted as cloud. In order to avoid this problem $T_{r}$ is adjusted by modelling the ground cooling effects at night in terms of the night length.

The procedure for cloud detection used observations made at both $6.7 \mu \mathrm{m}$ and $10.7 \mu \mathrm{m}$. First, the 6.7$\mu \mathrm{m}$ imagery is used to determine the existence of transparent or opaque cirrus at high altitude. If a pixel is determined to have opaque cirrus then the final cloud cover classification for that pixel location is "opaque" since the corresponding pixel in the 10.7- $\mu \mathrm{m}$ image would also give an opaque signature. However, if a pixel is either clear or transparent from the $6.7-\mu \mathrm{m}$ image analysis, the corresponding pixel in the $10.7-\mu \mathrm{m}$ image is examined. If cloud is detected in that pixel then the pixel location is classified as opaque. If not, then pixel locations classified respectively as clear or transparent remain clear or transparent.

The sky cover classifications described in the previous paragraph are the extent of those possible for individual pixels. A more accurate determination of clear (hence photometric) and partly cloudy (spectroscopic) conditions can be made using a cluster of pixels to represent the site instead of an individual pixel. As shown schematically in Figure 2, a 9-pixel area may be used to represent the astronomical "sky" at the site. At the level of the Tropopause (about $12 \mathrm{~km}$ ), for an observer on the ground viewing the sky, this 9-pixel area would correspond to the sky within approximately $62^{\circ}$ of zenith.

The number of pixels within the area for each cloud cover category was counted and that count used to provide a more accurate measure of observing conditions. For the 9-pixel area the following classification scheme of cloud cover and observing conditions was used:

Clear (Photometric): All 9 pixels are clear

Transitional (Spectroscopic): 6-8 pixels are clear (1-3 pixels are partially transparent or opaque)

Opaque (Unsuitable for astronomy): 5 or fewer pixels are clear

In order to verify the accuracy of cloud cover observations using the above methodology a comparison was made between the satellite and records of observing conditions at San Pedro Martir Observatory (SPMO) for a one year period (June 1997 to May 1998). Using periods of "half-nights", conditions were classified as Photometric (less than $15 \%$ cloud cover or no more than 30 minutes of cloud cover in a 5 hour period) or Spectroscopic (more than $15 \%$ but less than $65 \%$ cloud cover). ${ }^{11}$ These definitions are fairly similar to the satellite-based categories defined above. Figure 3 is a plot of the monthly values. In some months, for the 


\begin{tabular}{|c|c|c|}
\hline 2 & 3 & 4 \\
\hline 9 & Site & 5 \\
\hline 8 & 7 & 6 \\
\hline
\end{tabular}

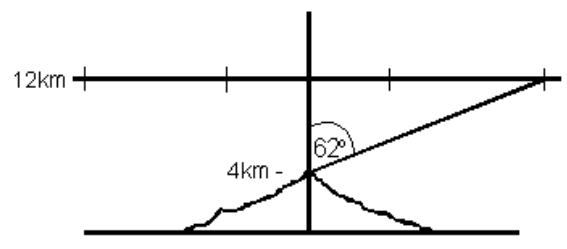

Figure 2. Schematic diagram showing the 9-pixel site area in plan view (left) and cross-section (right). At left, each square represents a $10 \mathrm{~km} \times 10 \mathrm{~km}$ pixel in the satellite image. At right, assuming a site altitude of $4 \mathrm{~km}$, at Tropopause level (approximately $12 \mathrm{~km}$ ), the "sky" encompassed by the 9-pixels corresponds approximately to an area of observation within $62^{\circ}$ of zenith.

ground-based observations, less than half of the nights were sampled. For the satellite, observations are made every night, typically three times per night. The annual figures for both photometric and spectroscopic fractions are shown in Table 1.

Table 1. Photometric and spectroscopic fractions (\%) for San Pedro Martir Observatory for the year June 1997 to May 1998 from satellite and ground-based observations.

\begin{tabular}{|l|l|l|l|}
\hline & Photometric & Spectroscopic & Useable \\
\hline Satellite & 69.8 & 11.8 & 81.6 \\
\hline Ground & 67.5 & 15.6 & 83.1 \\
\hline
\end{tabular}

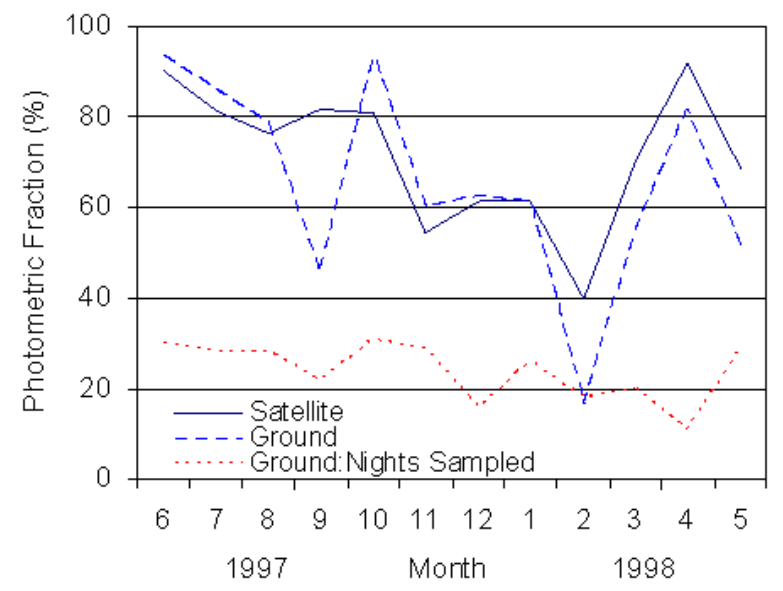

Figure 3. Photometric fraction by month at San Pedro Martir Observatory for the period June 1997 to May 1998 as determined from satellite and ground-based observations.

It is clear from this comparison that there is good agreement between satellite-based and ground-based measurements of observing conditions for San Pedro Martir Observatory. Differences in the fractions obtained for each observing method are consistent with differences in the definitions of what constitutes photometric or spectroscopic conditions. Similar levels of agreement were found at Paranal Observatory. ${ }^{5}$ 


\subsection{Results}

Using the methodology described above, cloud cover over the study area was quantified and mapped. In the aerial mapping, only cloud above the $800 \mathrm{mb}$ pressure level $(\approx 2000 \mathrm{~m})$ or the surface (if higher) is included. In determining the "surface" altitude, allowance is made for the fact that the digital terrain is smoothed and for the ground cooling effect described in Section 3.2. The net result is that cloud up to about $500 \mathrm{~m}$ above the ground (on average) goes undetected at night. Typically, this would lead to cloud being underestimated by $1-$ $2 \%^{5}$ in the area mapping.

Figures 4 and 5 show the clear (photometric) and clear plus transitional (useable) fractions respectively. The maps are for the period starting one hour after true local sunset time to one hour before true local sunrise time. The maps were compiled using the 9-pixel area (as defined in Section 3.2) centered on each pixel location in the study domain. The frequency of occurrence of each cloud cover category was then counted for each pixel location, expressed as a percentage frequency and then isokephs (lines joining places with equal frequency of occurrence) were drawn.

Cloud cover is a minimum in the subtropics $\left(28^{\circ} \mathrm{N}\right.$ to $\left.35^{\circ} \mathrm{N}\right)$ where high pressure prevails for most of the year. Cloudiness increases to the north of these latitudes due to the increasing frequency of winter storms (midlatitude cyclones) and to the south due to greater tropical storm (cyclones, depressions, monsoonal disturbances and local convection) activity in the summer. Over the land, there is also a general increase in cloudiness from west to east. This is consistent with the fact that, in the subtropics, high pressure (sinking air and clear skies) is a more permanent feature off the west coast of the continents. This is the case because cold ocean temperatures inhibit rising motion in the atmospheric boundary layer (surface to $1000 \mathrm{~m}$ ), allowing subsidence to prevail above this layer. On the other hand, areas east of the continental divide are influenced by strong surface heating over the land and moisture off the Gulf of Mexico. These conditions favor rising motion and cloud formation.

A large area with a clear fraction of $70 \%$ or more is observed over southern California, western Arizona and northern Baja, Mexico. In Baja this area is about $50 \mathrm{~km}$ wide extending along the spine of the peninsula northwards of $28^{\circ} \mathrm{N}$. Small pockets where the clear fraction reaches $75 \%$ are found in this region at $30^{\circ} \mathrm{N}$, $31^{\circ} \mathrm{N}$, and $32.5^{\circ} \mathrm{N}$. The area where the clear fraction exceeds $70 \%$ extends into the desert interior of Southern California. (Note: The sudden kink in the isokephs over the ocean at $30^{\circ} \mathrm{N}$ is due to a scarcity of rawinsonde data over the oceans. Land areas are unaffected by this problem.)

Westwards of the optimal area described above, a steep gradient of cloudiness is observed near the coast. Coastal cloud associated with fog and low cloud formation below the inversion layer extends inland. At night, the inland extent is determined by local circulation patterns and whether the terrain extends above the height of the inversion layer. As indicated in Figure 4 this cloud is generally confined to within $50 \mathrm{~km}$ of the coast at night.

Inland, a significant cloudiness increase is observed towards the east and north of the optimal area. For example, at about $33^{\circ} \mathrm{N}$, traversing Arizona from west $\left(115^{\circ} \mathrm{W}\right)$ to east $\left(109^{\circ} \mathrm{W}\right)$, there is a $20 \%$ decrease in the clear fraction. In general, north of the optimal area, cloud cover increases due to the increasing frequency of occurrence of winter storms. However, two pockets where the clear fraction exceeds $65 \%$ and $70 \%$ respectively are found in the southern Sierra Nevada.

Examination of the map (Figure 5) showing the useable fraction (clear plus transitional cloudiness) indicates the same general pattern as the clear fraction map. Over the areas that are generally clear (clear fraction $>$ $65 \%$ ), the transitional cloud fraction (not shown) varies only slightly, being about $10 \%$. An interesting exception is found just north of the optimal area in the Sierra Nevada range where the transitional fraction is higher (over $15 \%$ ). This is almost certainly due to ground being detected over the very high peaks in this area. Thus the clear fraction for sites in this area may be up to $5 \%$ higher than indicated in the clear fraction map.

\section{CELT ON-SITE MEASUREMENT PLANS}

CELT passed its conceptual design review in May of 2002 and UC and CIT are currently in the process of developing a memorandum of understanding for the proposed $3-4$ year detailed design and development study. 


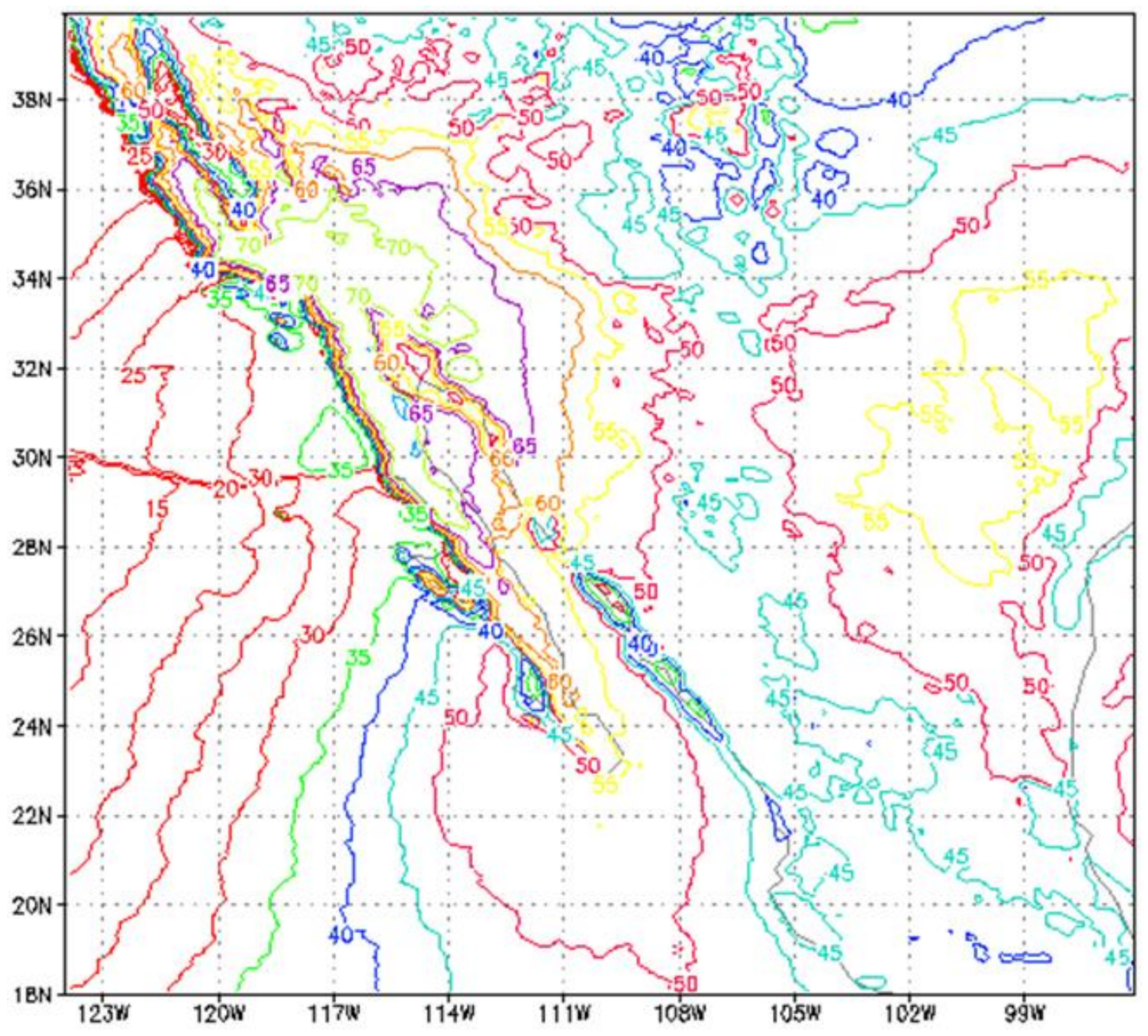

Figure 4. Clear fraction (\%) for the observing night over the 5-year study period.

While the site selection process is part of this detailed design phase, it was one of the recommendation of the review committee to pre-fund site selection, which is recognized as the critical pacing item for CELT at this point. As a result, some funding has been allocated recently and the first instruments for atmospheric turbulence characterization were ordered in August of 2002. The goal is to start on-site measurements in early 2003 and, eventually, to have 3 or 4 sets of instruments running simultaneously at different sites. We estimate that the total on-site characterization process will last between 3 and 4 years.

For telescopes up to a few meters in diameter, it is often sufficient to have knowledge of a few integrated parameters describing atmospheric turbulence, such as the atmospheric coherence length, $r_{0}$, the turbulence coherence time, $\tau_{0}$, and the isoplanatic angle, $\theta_{0} \cdot{ }^{12}$ As the telescope diameter increases, a more detailed description of turbulence above the site becomes necessary. Furthermore, as turbulence changes on all time scales, it is essential that the longest possible period of site characterization is covered. If one also wants to test more than one site, it quickly becomes clear that meaningful on-site testing for CELT can only be achieved with 


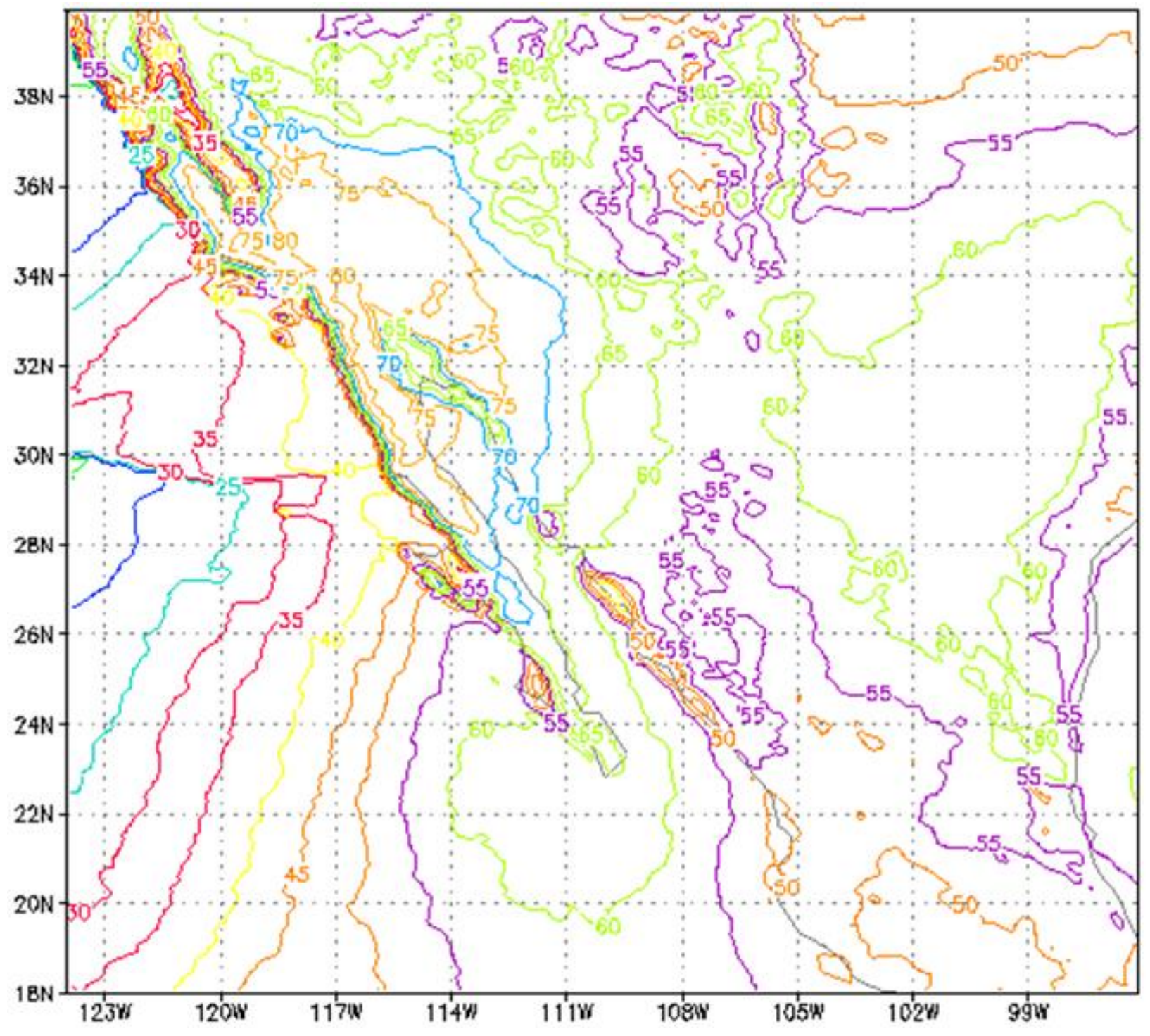

Figure 5. Useable (clear plus transitional cloudiness) fraction (\%) for the observing night over the study period.

automated robotic instruments which can run autonomously for extended amounts of time and which do not require frequent maintenance. Our plans concerning which parameters need to be measured - which is, obviously, always a compromise between all the quantities one would like to measure and what is feasible given the available means - and what instruments to use for these measurements are described in this section.

\subsection{Atmospheric Coherence Length, $r_{0}$}

For ELTs just as for smaller telescopes, the most important turbulence parameter is the atmospheric coherence length or Fried's parameter, $r_{0}$. The determination of $r_{0}$ will therefore be the core of our on-site measurements. One of the best established and most reliable instruments for measuring $r_{0}$ is the differential image motion monitor (DIMM) ${ }^{13}$ A DIMM measures the differential image motion between two small apertures (usually 5 $10 \mathrm{~cm}$ in diameter) which are separated by a distance on the order of a few times the individual aperture diameter. The strength of DIMMs is that telescope vibrations do not affect the measurements if the two apertures are 
part of one larger aperture or, to a slightly lesser degree of accuracy, if they are individual telescopes that are installed on the same mount.

The problem with most existing DIMMs is that one usually uses amateur telescopes in order to save cost in the instrument construction. This is feasible if the DIMMs are either used in campaign mode during short periods of time or if they are deployed at sites where frequent maintenance is available, such as established observatory sites. For CELT site testing, however, we need to set up our instruments at remote sites and have them operate for long periods of time without a person being present. The standard amateur telescope solution is therefore not feasible for us. Instead, our DIMMs will be using very robust mounts and telescopes that are based on the Very Large Telescope Interferometer siderostat design.

We note that the strategy described above, using automated and very reliable instruments, does not only apply to the DIMMs, but to all instruments which we are intending to use. While the instrument cost will be higher than it would be for less robust instruments, we expect that we will be saving money for personnel and maintenance with respect to solutions based on amateur telescopes and equipment.

\subsection{Distinction of the Ground Layer}

At most observatory sites, the largest contribution to the overall turbulence strength comes from the ground layer. This has two effects for CELT site testing. First, since it is not possible to elevate our DIMMs to the height at which the primary mirror of CELT will be located (approximately $30 \mathrm{~m}$ ), we need to be able to distinguish the turbulence that is located below the primary mirror from the above-the-mirror contributions. Second, if a strong ground layer up to approximately the elevation of the primary mirror exists, it is important to know if changing this elevation by a reasonable amount might change the overall turbulence seen by CELT significantly. As a result, it is indispensable, that the turbulence in the first approximately $40 \mathrm{~m}$ is measured separately. It can then be subtracted from the DIMM measurement, which is an integrated value of all turbulence located above the DIMM.

In order to measure the ground-layer turbulence, we will set up masts with micro-thermal probes next to our DIMMs. One problem of micro-thermal probes is that they only measure the turbulence strength at distinct points whereas an integrated value would be more useful. However, the interpolation between those points is considered reasonably reliable if a sufficiently small vertical spacing of the probes is used. We will nevertheless investigate the use of acoustic emitter-receiver systems for the mast which are, in theory, capable of measuring the integrated turbulence strength along the mast.

Even with the mast, it is still necessary to set up the DIMMs above the very lowest layers of turbulence so as to obtain the highest possible signal-to-noise ratio of the $r_{0}$ measurement. The DIMMs will therefore be set up on a platform of approximately 3 meters height at most sites. This height was chosen because it is a reasonable compromise between avoiding as much of the ground layer as possible and the technical problems of such a setup.

\subsection{Turbulence Profiles}

More generally than just for the distinction of the ground layer, obtaining vertical profiles of turbulence is important for ELTs. The reasons range from understanding the difference between individual locations on the same mountain, such as, for example, the difference between the northern shield and summit ridge locations on Mauna Kea, to optimizing the design and operation of multi-conjugate adaptive optics systems. In principle, if the exact profile of the turbulence strength, $C_{n}^{2}(h)$, and the wind velocity profile were known, almost all other quantities of interest for an ELT (at least as far as atmospheric turbulence is concerned) can be calculated from these two profiles. However, it is very difficult to measure the turbulence and wind profiles reliably. Only two methods, launching balloons with micro-thermal probes and anemometers and the SCIDAR method, ${ }^{14}$ exist which produce results with a sufficiently high resolution to determine all quantities needed for a complete characterization of turbulence. Neither of these methods is suitable for long-term characterizations of remote sites. We therefore intend to use a combination of different instruments that give us a variety of information about the turbulence profile. 
A relatively new concept of measuring turbulence profiles is the multi-aperture scintillation sensor, MASS. ${ }^{15}$ It uses differential scintillation measurements between 4 small concentric apertures $(2-8 \mathrm{~cm}$ in diameter $)$ to obtain low-resolution (approximately 6 points throughout the atmosphere) turbulence profiles. One of the advantages of the MASS profiler is that it can easily be implemented at the same telescope as a DIMM. Development of such an combined implementation is under way in collaboration with the Cerro Tololo InterAmerican Observatory, CTIO, and we expect to add MASS profilers to our DIMMs soon after the DIMMs become operational.

In addition to obtaining low-resolution profiles, we also want to understand the first few hundred meters of the turbulence profile with a much higher resolution than what is provided by the MASS profiler. One of the possible solutions to access this part of the profile is by using so-called SODARs, which stands for SOund Detection And Ranging. SODARs emit sound pulses which are scattered back by turbulent atmospheric layers. The back-scattered echo can be measured with sufficient signal-to-noise ratio from layers up to several hundreds of meters above the instrument. If several receivers are used, not only the turbulence profile but also the wind velocity profile can be measured with a vertical resolution of a few tens of meters.

An alternative method of accessing the lowest part of the turbulence profile is the flying of kites equipped with micro-thermal probes and anemometers. The advantage of kites is that they usually produce more accurate results than SODARs. The disadvantage is that many fewer profiles can be obtained. Both methods of accessing the low turbulence-profile are currently under consideration for CELT. It is also possible that we will occasionally launch balloons.

\subsection{Atmospheric Coherence Time, $\tau_{0}$}

The atmospheric coherence time, $\tau_{0}$ is important in that it determines the temporal bandwidth at which an adaptive optics system has to operate. Not only do we therefore want to find a site with low turbulence strength (large $r_{0}$ ), but we are also interested in slow turbulence (large $\tau_{0}$ ). However, $\tau_{0}$ is directly proportional to $r_{0}$ and maximizing $r_{0}$ already provides for a large coherence time. Since $\tau_{0}$ is another quantity that is not easily measured, we therefore will not put significant effort into the coherence time measurements. We are, however, working on modifying our DIMMs in such a way that they take a continuous time series of data at a high frame rate [most current DIMMs do not produce a continuous time series of measurements]. An (at least approximate) deduction of $\tau_{0}$ should therefore be possible from the DIMM data. Furthermore, $\tau_{0}$ can also be calculated from the $C_{n}^{2}$ and wind profiles.

\subsection{Other Atmospheric Parameters}

The isoplanatic angle, $\theta_{0}$, which can be used as a measure of the variation of the adaptive optics correction at different positions in the field of view, can be calculated from the $C_{n}^{2}$ profile. The crude profiles obtained with the MASS profiler are sufficient for a characterization of $\theta_{0}$ for CELT site selection purposes.

Another parameter that does, in principle, play a role for ELTs is the outer scale of turbulence, $\mathcal{L}_{0}$, which is approximately the scale length at which turbulence becomes anisotropic. $\mathcal{L}_{0}$ is difficult to measure with any kind of instrument and there exist no physical models — only ad-hoc mathematical models — which describe the behavior of the turbulence power spectrum for scale lengths larger than $\mathcal{L}_{0}$. Measurements of the outer scale using the Generalized Seeing Monitor [one of the few instruments that is sensitive to $\mathcal{L}_{0}$, under the assumption that the turbulence power spectrum is described accurately by a von Kármán spectrum] ${ }^{16}$ indicate that the outer scale is of similar magnitude (tens of meters) at most if not all observatory sites. Since small changes of $\mathcal{L}_{0}$ have a negligible impact on the design of adaptive optics systems and, in addition, the CELT adaptive optics system will likely have to be designed for the worst-case scenario (infinite outer scale) anyway, $\mathcal{L}_{0}$ will not be measured in the framework of the CELT site selection process

\subsection{Other Instruments, Overall Setup, and Logistics}

Two more instruments will be a part of a full CELT site evaluation setup. They have not yet been described in this section because they measure weather instead of turbulence parameters. The first kind of instrument, weather stations, will, of course, be deployed at all sites of interest. In fact, weather stations will likely 


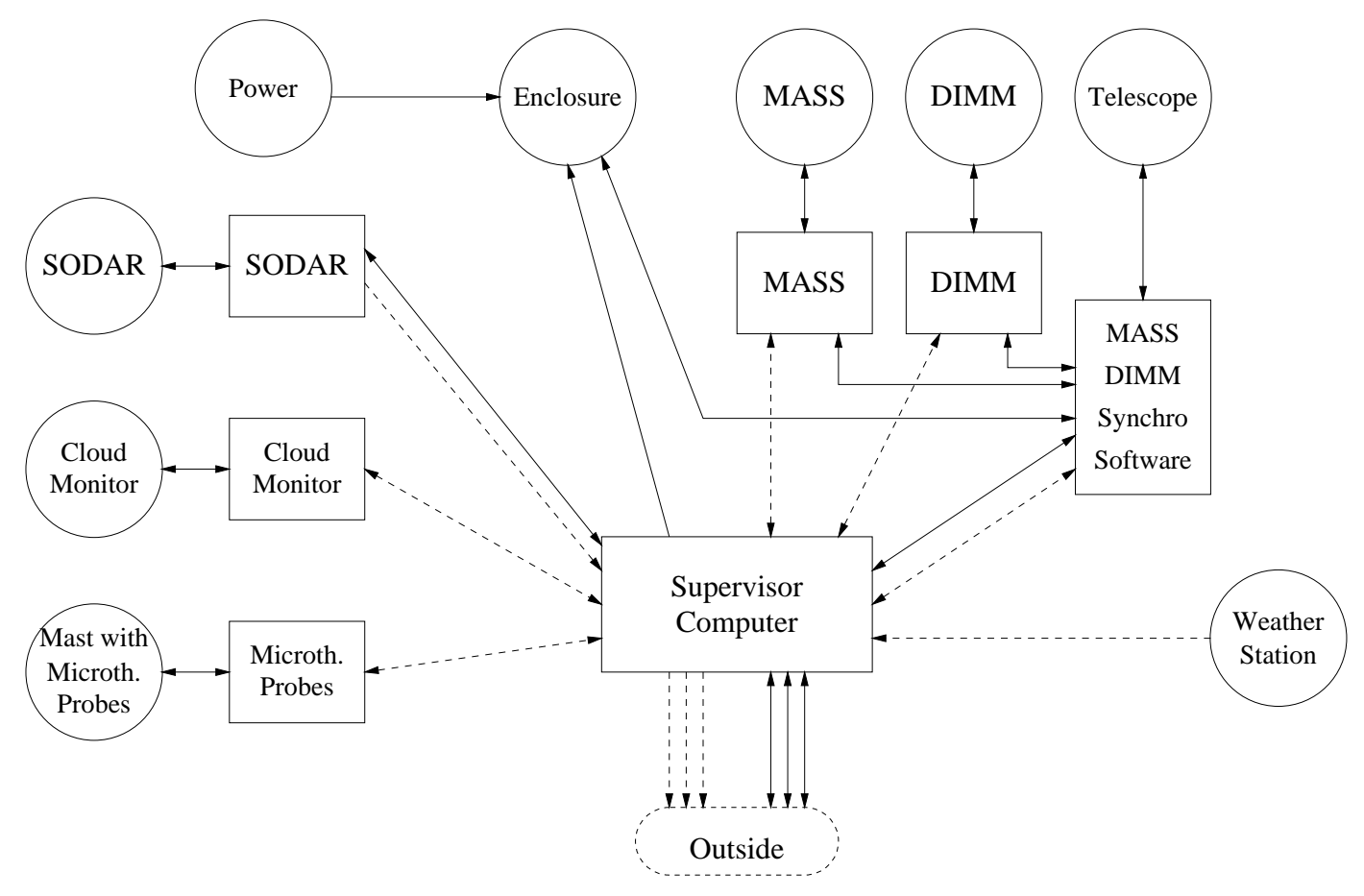

Figure 6. Representation of the logical (not the physical) setup of a potential complete CELT site evaluation set. Circles indicate physical instruments. Square boxes symbolize computers and/or software packages. Solid lines show connections between instruments that necessitate an exchange of commands, while dashed lines stand for connections for data downor uploads.

be operating at the sites before the first turbulence characterization equipment arrives. The other kinds of equipment are automated cloud cover and atmospheric transparency monitors. Such monitors might be useful for remote sites, while they are certainly not needed at or near established observatory sites.

Figure 6 is a representation of the logical (not the physical) setup of a potential complete CELT site evaluation set. Circles indicate physical instruments. Square boxes symbolize computers and/or software packages. Solid lines show connections between instruments that necessitate an exchange of commands, while dashed lines stand for connections for data down- or uploads. We will not go into the details of Fig. 6 in this paper, but use it only to point out that the reliable robotic operation of such a setup is not a trivial matter. We will therefore start our on-site campaigns with a reduced setup consisting of only the DIMM [with telescope and enclosure], weather station, mast with microthermal probes, supervisor computer, and power supply. Once this initial setup is working reliably, we will then add instruments piece by piece until the complete setup is working as desired.

To conclude this section, we point out that many logistical issues need to be considered for installing and operating a setup of the magnitude shown in Fig. 6. At remote sites the supply of power and Internet connections is not a trivial issue and roads to the site might not exist. Another problem is the protection from lightning and snow storms, as sites are generally located on the tops of mountains. Finally, issues such as shelters for the setup and maintenance crews, spare parts and computers, and tools need to taken into account.

\section{SUMMARY}

We have presented the current status of the CELT site selection project. Satellite studies of cloud cover and precipitable water vapor have been performed for northern Chile and southwestern North America. Here we describe the North America study as an example. We will use these studies for a pre-selection of sites on which 
in-depth on-site testing will be performed. The on-site testing will be centered around the characterization of atmospheric turbulence and should start in early 2003. We have described which quantities we intend to measure and what instruments will be used for this purpose.

\section{ACKNOWLEDGMENTS}

The CELT site selection project has benefitted greatly from our collaboration with with the Giant Segmented Mirror Telescope (GSMT) site selection group of the National Optical Astronomy Observatory (NOAO), in particular with Alistair Walker, Robert Blum, Hugo Schwarz, and Andrei Tokovinin. We also want to thank Marc Sarazin from European Southern Observatory (ESO) for many useful discussions.

This work has been supported in part by the National Science Foundation Science and Technology Center for Adaptive Optics, managed by the University of California at Santa Cruz under cooperative agreement No. AST-9876783.

\section{REFERENCES}

1. "California Extremely Large Telescope, Conceptual Design of a Thirty-Meter Telescope," CELT Report Number 34, University of California, California Institute of Technology, June 2002.

2. J. E. Nelson, "Design concepts for the California Extremely Large Telescope (CELT)," in Proc. SPIE Vol. 4004, p. 282-289, Telescope Structures, Enclosures, Controls, Assembly/Integration/Validation, and Commissioning, Thomas A. Sebring; Torben Andersen; Eds., 4004, pp. 282-289, Aug. 2000.

3. J. E. Nelson, "CELT Rocks," in Proc. SPIE Vol. 4840, Future Giant Telescopes, 4840, 2002.

4. CELT website: http://celt.ucolick.org/.

5. D. Erasmus and C. van Staden, "A Satellite Survey of Water Vapor and Cloud Cover in Northern Chile," final report, Cerro-Tololo Inter-American Observatory, 2001.

6. D. Erasmus, "A Satellite Survey of Water Vapor and Cloud Cover at Selected Existing and Potential Infrared Telescope Sites in the South-western U.S.A.," final report, Rocky Mountain Observatories Consortium, Dec. 2000.

7. B. J. Soden and F. P. Bretherton, "Upper tropospheric relative humidity from the GOES $6.7 \mu \mathrm{m}$ channel: Method and climatology for July 1987," J. Geophys. Res. 98, pp. 16669-16688, Sept. 1993.

8. B. J. Soden and F. P. Bretherton, "Interpretation of TOVS water vapor radiances in terms of layer-average relative humidities: Method and climatology for the upper, middle, and lower troposphere," J. Geophys. Res. 101, pp. 9333-9344, Apr. 1996.

9. D. Erasmus and M. Sarazin, "Forecasting Precipitable Water Vapor and Cirrus Cloud Cover For Astronomical Observatories: Satellite image processing guided by synoptic model dissemination data," in Proc. SPIE Vol. 4168, Image and Signal Processing for Remote Sensing IV, 4168, Sept. 2000.

10. D. Erasmus and M. Sarazin, "Utilizing Satellite Data For Evaluation and Forecasting Applications At Astronomical Sites," in IAU SITE2000 Conference: Astronomical Site Evaluation in the Visible and Radio Range. Marrakesh, Morocco, 13-17 November, 2000, Nov. 2000.

11. M. Tapia, "Ten Years of Weather and Observing Statistics in San Pedro Martir, Baja California, Mexico," Revista Mexicana de Astronomia y Astrofisica 24, p. 179, Oct. 1992.

12. J. W. Hardy, Adaptive optics for astronomical telescopes, Oxford University Press, 1998.

13. F. Roddier and M. Sarazin, "The ESO differential image motion monitor," $A \mathscr{E} A$ 270, pp. 294-300, 1990.

14. A. Fuchs, M. Tallon, and J. Vernin, "Focusing on a turbulent layer: Principle of the "Generalized SCIDAR"," PASP 110, pp. 86-91, 1998.

15. V. Kornilov, A. Tokovinin, O. Vozyakova, A. Zaitsev, N. Shatsky, S. Potanin, and M. Sarazin, "MASS: a monitor of the vertical turbulence distribution," in Proc. SPIE Vol. 4839, Adaptive Optical System Technologies II (Peter L. Wizinowich, Domenico Bonaccini; Eds., 4839, 2002.

16. A. Ziad, R. Conan, A. Tokovinin, F. Martin, and J. Borgnino, "From the Grating Scale Monitor to the Generalized Seeing Monitor," Appl. Opt. 39, pp. 5415-5425, Oct. 2000. 